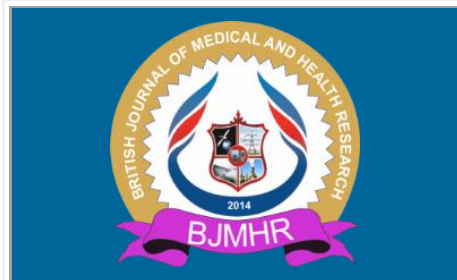

\title{
BJMHR
}

British Journal of Medical and Health Research

Journal home page: www.bjmhr.com

\section{Signet Ring Carcinoma of the Appendix- A Rare Case Report}

\author{
Kafil Akhtar*, Suhailur Rahman, Kiran Alam, Afzal Anees \\ Jawaharlal Nehru Medical College, Faculty of Medicine, Aligarh Muslim University, \\ Aligarh, UP-India.
}

\section{ABSTRACT}

Primary signet-ring cell carcinoma (SRCC) of vermiform appendix is extremely rare. A 62year-old man presented to the Surgical Clinics with right lower abdominal pain, where he was diagnosed with acute appendicitis based on the history and examination with hematologic investigations and computed tomography. Appendectomy specimen grossly showed acute inflammation with appendiceal adhesions and body of the appendix showed wall thickening and luminal occlusion. Microscopically nests of carcinoma cells were seen with compressed nuclei and intracytoplasmic mucin invading into the muscular layer. No lymphovascular permeation was seen. The cut margins were negative for carcinoma cells. Immunohistochemically, SRCC cells were positive for cytokeratin (CK) AE1/3, EMA, CEA, MUC2, and high Ki-67 labeling index. They were negative for CK5/6, CK7, Vimentin, MUC1 and chromogranin. Our patient is fine after 6 months of follow up, with no evidence of recurrence or metastasis.

Keywords: Appendix, Signet Ring, Carcinoma, Histopathology, Immunohistochemistry. 


\section{INTRODUCTION}

Appendiceal cancer is very rare and accounts for only $0.5 \%$ of all gastrointestinal neoplasms. ${ }^{1}$ According to a nationwide cancer database (SEER), the age-adjusted incidence of appendiceal malignancies was 0.12 cases per $1,000,000$ people per year. ${ }^{1}$ The majority of appendiceal tumors are neuroendocrine neoplasms (50-75\%), while most of the remaining tumors are adenocarcinomas. $^{2}$

Primary appendiceal cancer is diagnosed in only $0.9 \%-1.4 \%$ of appendectomy specimens. ${ }^{3}$ It looks like acute appendicitis and it is difficult to diagnose it on clinical grounds alone. The diagnosis is always confirmed by histopathology of a surgically removed appendix. Further, signet-ring cell carcinoma (SRCC) of vermiform appendix is extremely rare, accounting for $0.43 \%$ of all appendiceal malignancies. ${ }^{4}$ If signet ring cells account for more than $50 \%$ of the neoplasms, the term "signet ring carcinoma" is appropriate. Such lesions should be included in the high-grade category. Primary signet ring cell carcinoma is a rare event in surgery. ${ }^{5}$ More often, it presents as acute appendicitis, so it is hard to diagnose it on clinical grounds alone, and the diagnosis is always confirmed by histopathology of a surgically removed appendix. ${ }^{6-8}$ Pre-operative diagnosis of this tumor can be confusing because it does not show specific clinico-radiological signs and can be misinterpreted as an inflammatory process. We report a very rare case of primary appendiceal SRCC clinically and pathologically presenting as typical acute appendicitis.

\section{CASE SUMMARY}

A 62-year-old man presented to the Surgical Clinics with right lower abdominal pain, where he was diagnosed with acute appendicitis after physical examination, hematologic and radiographic investigations. Computed tomography demonstrated appendiceal swelling. Physical examination showed tenderness in the right iliac fossa with Blunberg sign and Rosenstein sign. Blood test showed leukocytosis with counts of 13,200/dl and predominant bilobed neutrophils with toxic granulations and increased C-reactive protein of $2.24 \mathrm{mg} / \mathrm{dl}$. Appendectomy was performed. Gross examination showed an inflamed appendix with appendiceal adhesion and acute peritonitis with no apparent tumor, but the body showed wall thickness of $1.1 \mathrm{~cm}$ with luminal narrowing (Figure 1). Microscopically nests of signet ring carcinoma cells with eccentric nuclei were seen, invading into the muscular layer (Figure 2 and 3). No lympho-vascular permeation was seen. The cut margins were negative for carcinoma cells. No distant metastasis, lymph node metastasis or peritoneal dissemination was found by imaging techniques as well as by clinical findings. The TNM stage of the appendiceal carcinoma was given as pT2N0M0. 


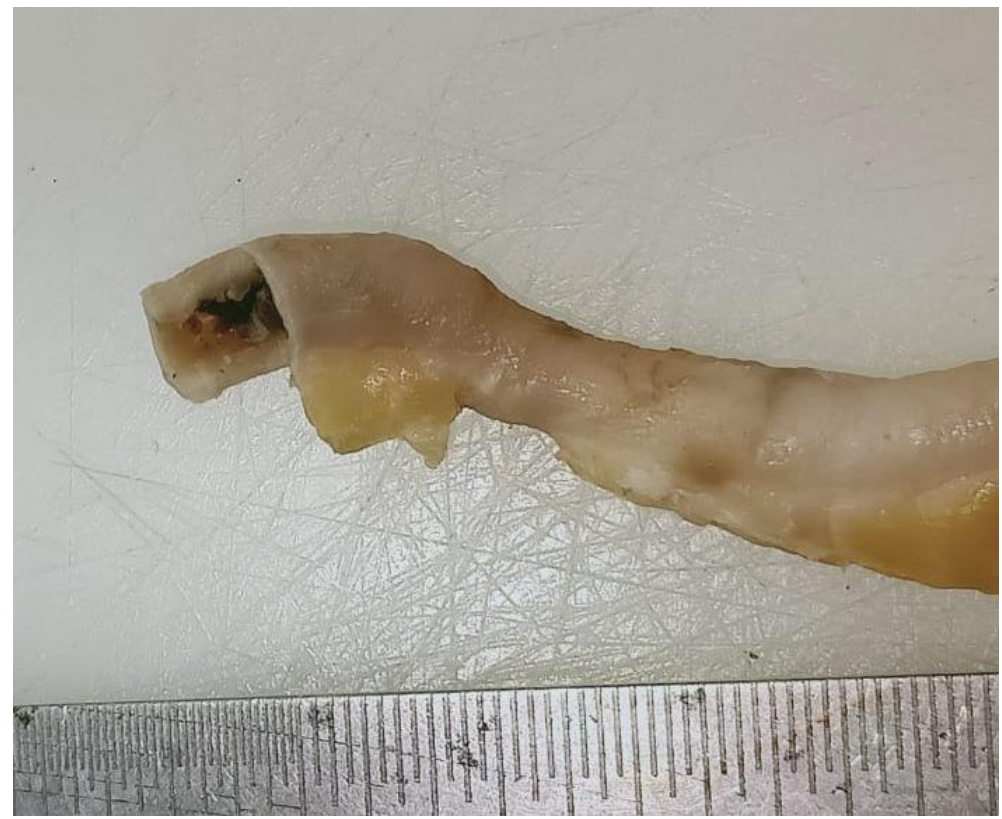

Figure 1: Gross examination showed an inflamed appendix with appendiceal adhesion the body shows wall thickening and luminal narrowing.

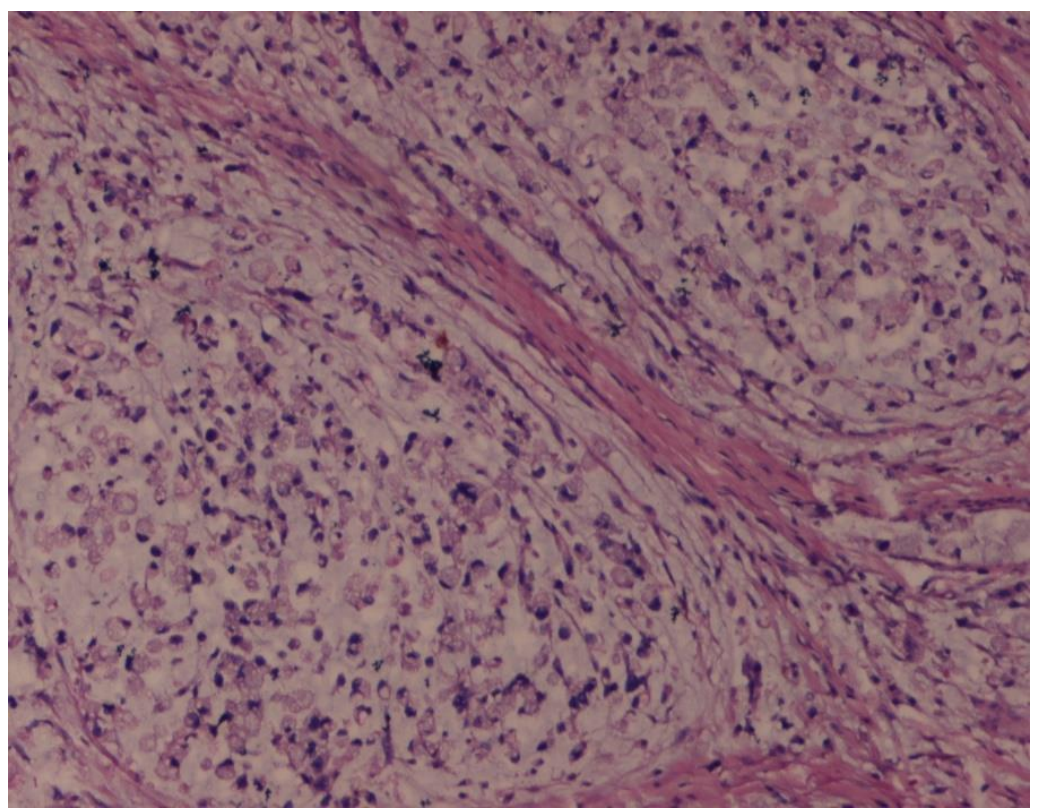

Figure 2: Microscopically nests of signet ring carcinoma cells were seen, invading into the muscular layer. Hematoxylin and Eosin x100. 


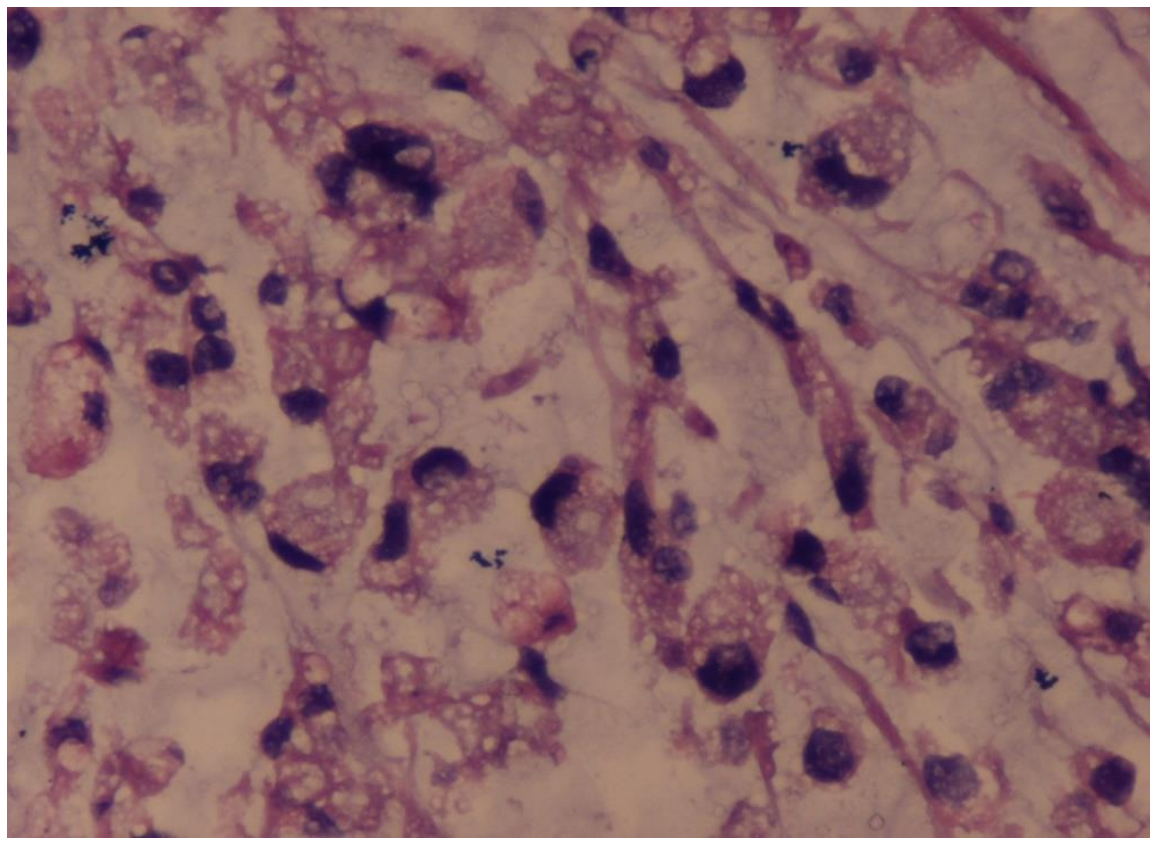

Figure 3: High power of Figure 2. Hematoxylin and Eosin x400.

Immunohistochemically, the SRCC cells were positive for cytokeratin (CK) AE1/3 (Figure 4), CK20, EMA, CEA, CDX2, MUC2 with high KI-67 labeling index. They were negative for CK7, MUC1, Vimentin and chromogranin (Figure 5). No further therapeutic treatments were performed, because the appendiceal carcinoma was small and the surgical margins were negative. Our patient is doing well after 6 months of follow up, with no evidence of recurrence or metastasis.

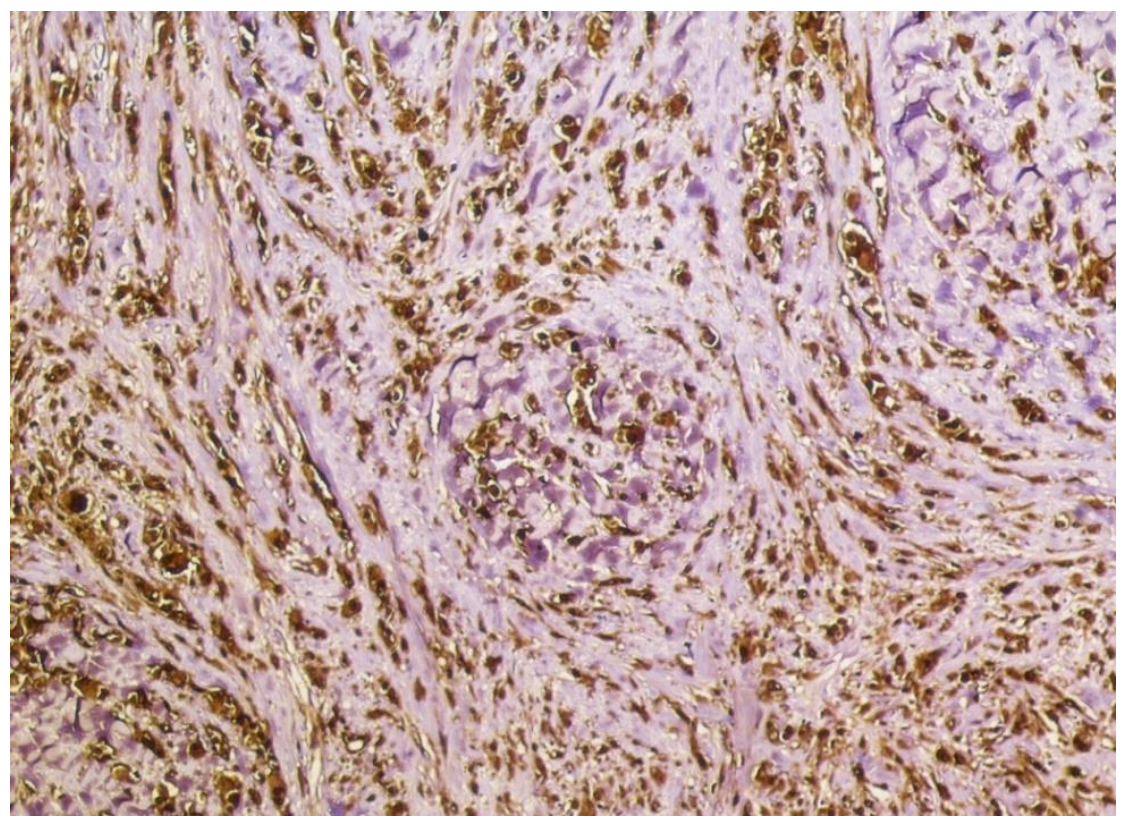

Figure 4: Immunohistochemically, the SRCC cells showed intense cytoplasmic positivity for cytokeratin (CK) AE1/3. IHC CK x100. 


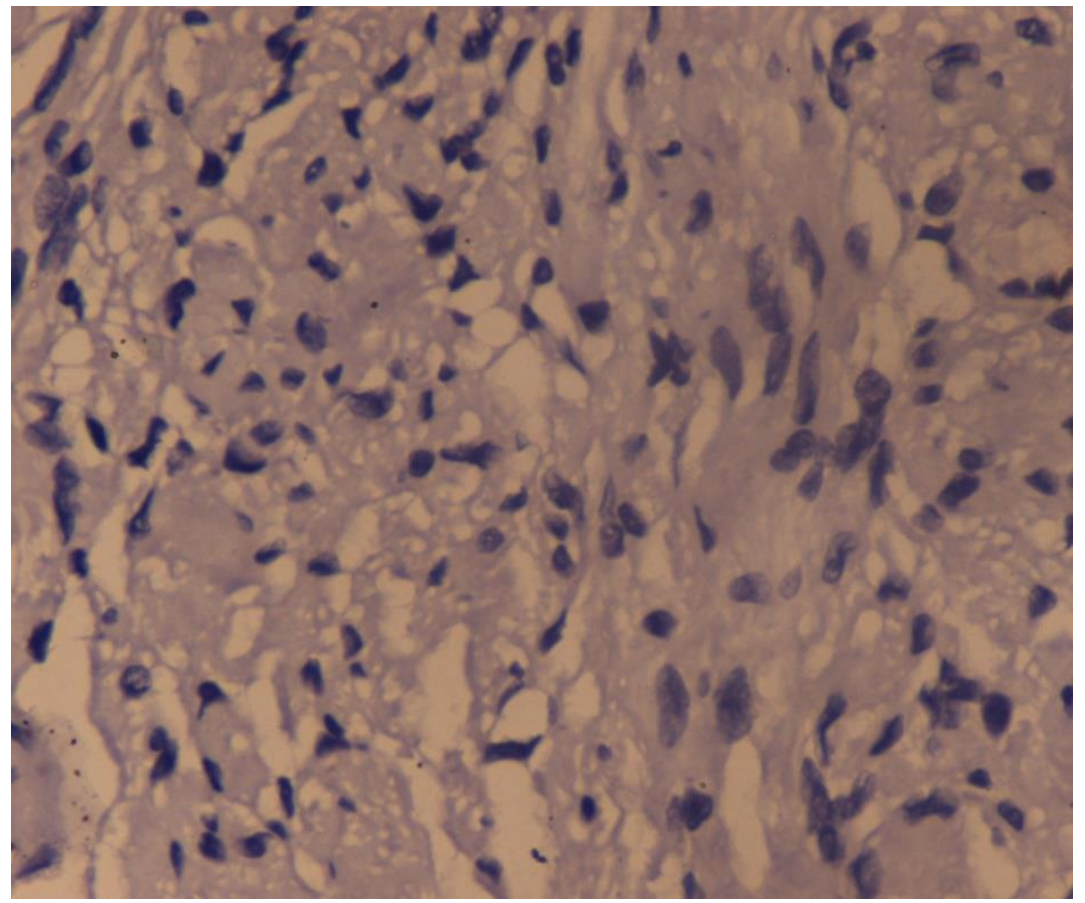

Figure 5: Immunohistochemically, the SRCC cells were negative for Chromogranin. IHC Chromogranin x400.

\section{DISCUSSION}

Non-neuroendocrine appendiceal malignancies are very rare and represent less than $0.5 \%$ of all gastrointestinal malignancies. ${ }^{3}$ The prevalence rate in the literature is around $0.3 \% .^{6}$ Adenocarcinomas of the appendix are less common than appendiceal neuroendocrine tumors, and since the first case was reported by Berger in 1882, there have been only a few hundred cases described in the literature. ${ }^{1}$

The primary SRCC of the current case was very small and the incidental finding of the appendectomy. This suggests that pathologist should perform meticulous examination of the appendectomies. The mean age at presentation is 63 years and, with men are more often affected than women. ${ }^{9}$ Signet ring carcinomas are even rarer then adenocarcinomas and they constitute only $4 \%$ of all neoplasms of the appendix. ${ }^{6}$

The present SRCC of the appendix seems to be the primary site since no tumors other than the appendiceal tumor were found in the body by various imaging modalities, and also because the SRCC cells of the present tumor were immunohistochemically positive for CDX2, a colonic type epithelium-specific antigen. ${ }^{10}$ Interestingly, the present primary SRCC of appendix clinically manifested as acute appendicitis. The physical findings, blood test, and CT findings were typical for acute phlegmonous appendicitis with focal wall thickening in the body of appendix. The tumor invaded the muscular layer (pT2), and no lymphovascular permeation was seen with TNM stage of pT2N0M0. The prognosis of this patient was not bad, although histological type (SRCC) are notable for poor prognosis. Our patient is free from recurrence and metastasis, 6 months after the operation. 
An extensive immunohistochemical study of primary appendiceal SRCC was performed. The $\mathrm{CK}$ profile of $\mathrm{CK} 7-/ \mathrm{CK} 20+$ pattern is compatible with appendiceal origin. EMA was expressed and vimentin was not expressed, suggesting that the present tumor is epithelial origin. Ki-67 labeling was high, suggesting a high cell proliferative activity. The present SRCC expressed CEA, indicating that the present tumor is a kind of adenocarcinoma. The MUC profiles suggest that product of MUC2 gene is operative, while MUC1 gene is not. The negative reaction for chromogranin indicates that the present tumor is not goblet cell carcinoid.

On CT imaging, neoplastic processes are more likely associated with wall thickening that is more than $3 \mathrm{~cm}$, involvement of a short segment, and/or an abrupt transition point. ${ }^{11}$ Heterogeneous attenuation is more common in ischemia and necrosis and seen in poorly differentiated gastrointestinal tumors. Fat stranding is more commonly associated with inflammatory conditions, while asymmetrical/eccentric wall thickening is associated with malignant processes. ${ }^{12}$

Signet ring carcinomas are considerably more likely to have extended at the time of diagnosis, as compared to other primary appendiceal tumors. ${ }^{13,14}$ Essentially, lymphatic-space metastases can closely mimic inflammatory conditions. ${ }^{15,16}$ The majority of the appendix carcinomas are identified accidentally, and many are advanced at the time of diagnosis. ${ }^{17}$ The extent of the tumor at the time of the diagnosis is an important determinant of the patient's future prognosis. It has a very poor prognosis, with a 5-year survival of $7 \% .{ }^{18,19}$ Median disease-specific survival is 25 months. ${ }^{20}$

\section{CONCLUSION}

Scrupulous histopathological examination of the appendix is an obligatory procedure. Elimination of signet ring cell carcinoma from other carcinoma subtypes is of special importance as it has an exceptionally poor prognosis and is generally diagnosed in advanced stages.

\section{REFERENCES}

1. McCuster ME, Cote TR, Clegg LX, Sobin LH. Primary malignant neoplasms of the appendix: a population-based study from the surveillance, epidemiology and endresults program, 1973-1998. Cancer 2002; 94:3307-3312.

2. Behera PK and Rath PK. Primary appendiceal Mucinous Aden carcinoma. Indian J Surg 2009;68:1221-1225.

3. O’Donnell ME, Badger SA, Beattie GC, Carson J, Garstin WI. Malignant neoplasms of the appendix. Int J Colorectal Dis 2007; 22:1239-1248. 
4. Suzuki J, Kazama S, Kitayama J, Uozaki H, Miyata T, Nagawa H. Signet ring cell carcinoma of the appendix manifesting as colonic obstruction and ovarian tumors: report of a case. Surg Today 2009; 39: 235-240.

5. Ho Ko Y, Jung CK, Oh SN, Kim TH, Won HS, Kang JH et al. Primary signet ring cell carcinoma of the appendix: a rare case report and our 18-year experience. World $\mathbf{J}$ Gastroenterol 2008;14: 5763-5768.

6. Mastoraki A, Papanikolaou IS, Kanakis D, Safioleas P, Sakorafas G, Safioleas M. A case of signet ring cell carcinoma of the appendix: dilemma in differential diagnosis and management. J Gastrointest Cancer 2010; 41:141-144.

7. Terada T, Kawaguchi M, Furukawa K, Sekido Y, Osamura Y. Minute mixed ductalendocrine carcinoma of the pancreas with predominant intraductal growth. Pathol Int $2002 ; 52: 740-746$.

8. Jonatan V, Pavle V, Cindrob S, Tomica C, Ante T. Signet Ring Carcinoma of the Appendix Presenting as Crohn's Disease in a Young Male. Case Rep Gastroenterol 2018;12:277-285.

9. Katelynn D, Andrew MB, Carlos HF, Chan L. An Unusual Progression of Signet-Ring Cell Carcinoma of the Appendix in a Caucasian Woman. J Gastrointest Canc 2019; 50:331-333.

10. Carr NJ and Sobin LH. Adenocarcinoma of appendix. In: Bosman FT, Car neiro F, Hruban RH, Theise ND, editors. WHO Classification of tumours of the digestive system. Lyon: IARC; 2010. p. 122-5.

11. Filik M, Kir KM, Aksel B. The role of $18 \mathrm{~F}-\mathrm{FDG}$ PET/CT in the primary staging of gastric cancer. Mol Imaging Radionucl Ther 2015; 24:15-20.

12. Ha TK, Choi YY, Song SY. F18-fluorodeoxyglucose-positron emission tomography and computed tomography is not accurate in preoperative staging of gastric cancer. $\mathbf{J}$ Korean Surg Soc 2011;81:104-110.

13. Terada T. Primary signet-ring cell carcinoma of vermiform appendix clinically and pathologically presenting as acute appendicitis. Human Path Case Reports 2014; 1: 69.

14. Hashash JG, Nasr JY, Francis F. Education and Imaging. Gastrointestinal: mucinous and signet cell adenocarcinoma of the appendix. J Gastroenterol Hepatol 2013; 28(6):903.

15. Hristova L, Soyer P, Hoeffel C, Marteau P, Oussalah A, Lavergne-Slove A et al. Colorectal cancer in inflammatory bowel diseases: CT features with pathological correlation. Abdom Imaging 2013; 38(3):421-435. 
16. Murdock T, Lim N, Zenali M. Lymphangitic spread from the appendiceal adenocarcinoma to the ileocecal valve, mimicking Crohn's disease. World J Gastroenterol 2015; 21(7):2206-2209.

17. Kulkarni RV, Ingle SB, Siddiqui S. Primary signet ring cell carcinoma of the appendix: a rare case report. World J Clin Cases 2015; 3:538-341.

18. Khan SA, Dwyer JP, Vaughan R. Peritoneal carcinomatosis from signet ring cell adenocarcinoma of the appendix. Gastrointest Endosc 2017; 86:569-571.

19. Wu SG, Chen XT, Zhang WW. Survival in signet ring cell carcinoma varies based on primary tumor location: a surveillance, epidemiology, and end results database analysis. Expert Rev Gastroenterol Hepatol 2018; 12:209-214.

20. Erçetin C, Dural AC, Özdenkaya Y. Metastatic gastric signet-ring cell carcinoma: a rare cause of acute appendicitis. Ulus Cerrahi Derg 2015; 32:140-144.

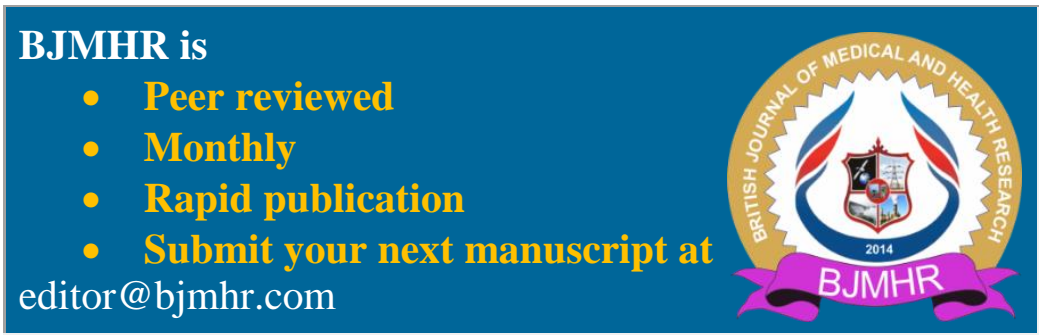

